\title{
Clinical diagnosis and computer analysis of headache symptoms
}

\author{
PD DRUMMOND, JW LANCE \\ From the Department of Neurology, The Prince Henry Hospital, and the School of Medicine, University of \\ New South Wales, Australia
}

SUMMARY The headache histories obtained from clinical interviews of 600 patients were analysed by computer to see whether patients could be separated systematically into clinical categories and to see whether sets of symptoms commonly reported together differed in distribution among the categories. The computer classification procedure assigned 537 patients to the same category as their clinical diagnosis, the majority of discrepancies between clinical and computer classifications involving common migraine, tension-vascular and tension headache. Cluster headache emerged as a clearly-definable syndrome, and neurological symptoms during headache were most prevalent in the classical migraine group. However, the classical migraine, common migraine, tensionvascular and tension headache categories differed in terms of the number, rather than the nature, of common migraine features. Whether the two extremes of this migraine-tension headache spectrum are different disorders can be determined only by studies of their pathophysiology.

It has recently been argued that clinically-accepted definitions of recurrent headache syndromes lack the precision required to delineate homogeneous subgroups of patients. ${ }^{1}$ To see whether clinical diagnoses were being made objectively, Diehr et $a^{2}$ compared the clinical classification of patients with symptoms of migraine or of tension headache with the results of a computer classification procedure. Eighty-nine per cent of the tension headache group and $88 \%$ of migrainous patients were assigned by the computer to their clinical category, indicating that some objective criteria were being used in making these two headache diagnoses. Nevertheless, more than $30 \%$ of patients were not assigned a diagnosis by the clinician, apparently because symptoms were intermediate between migraine and tension headache.

To assess the objectivity of common headache diagnoses, each patient in the present series was assigned by clinical analysis ${ }^{3}$ to one of five categories (classical migraine, common migraine, tensionvascular or "mixed" headache, tension headache, and cluster headache). An objective computerised

Address for reprint requests: Professor JW Lance, The Prince Henry Hospital, Little Bay, 2036 New South Wales, Australia.

Received 21 February 1983 and in revised form 29 August 1983. Accepted 12 September 1983 classification procedure (stepwise discriminant analysis) was then used to assign patients to the clinical category which their headache pattern resembled most closely. Each patient's computer classification was compared with his or her clinical diagnosis.

The difficulty associated with defining recurrent headache syndromes has stimulated a search for natural sets of headache characteristics not based on clinical preconceptions. Several attempts to define headache syndromes by factor analysis, a correlational technique which groups variables sharing something in common, have met with little success. ${ }^{4-6}$ The most striking finding has been the absence of a factor containing the variables: unilateral headache, gastrointestinal disturbance and focal neurological symptoms. Thus three of the cardinal features used to define migraine apparently were reported as often by themselves as in specific combinations.

One of the difficulties of interpreting the results of factor analyses of headache characteristics has been the large number of factors identified. For example, a recent factor analysis of 49 headache features ${ }^{5}$ resulted in 17 statistically-independent factors, although some of these factors appeared to be a natural consequence of the variables included in the symptom checklist. Several factors pointed to an 
association between the same or very similar symptoms before, during and after headache, while other factors resulted from asking the same question twice (the most notable example was a factor which indicated that unilateral headache was not associated with pain on both sides of the head). In the present investigation, we tried to obtain a simpler factor solution by screening variables for redundancy and by restricting the symptom checklist to indices of headache frequency, site and symptoms associated with headache.

The final aim was to see whether the sets of headache characteristics identified by factor analysis would differ from one clinical category to another. To do this, a score was computed for each patient on each factor and these scores were compared statistically among the five clinical categories.

\section{Methods}

Six hundred patients referred to the neurology clinic or hospital service because of headache were interviewed by the authors or by neurological staff under the supervision of the authors. Relevant details were recorded on a standard headache history form. The clinical interview was supplemented by a neurological and general physical examination and, when indicated, laboratory tests to ensure that the headache was not caused by a spaceoccupying lesion or other structural cause.

During the clinical interview, the patient was asked about the frequency, duration and site of the headache, and the nature of symptoms preceding and accompanying headache (Appendix). Premonitory symptoms (mood swings such as elation, irritability or depression; hunger, particularly a craving for sweet food; thirst or drowsiness) which preceded the headache by up to 24 hours were recorded separately from prodromal symptoms (visual disturbance, paraesthesiae or other focal neurological symptoms during the hour or so preceding the headache). All items on the standard headache history form were marked as positive or negative and the details of positive findings recorded. Because some patients reported that headaches occasionally were unilateral but were bilateral at other times, unilateral and bilateral headaches were coded as two separate variables. A similar approach was used for presence or absence of nausea, vomiting, throbbing headache, and facial pallor or flushing. It was not possible to estimate accurately the grouping of symptoms during individual episodes of headache; instead, variables were coded as 1 or 0 depending on whether they were or were not associated with the patient's most severe headaches. Each headache history was reviewed by one of us (JWL) who assigned one of five clinical diagnoses (classical migraine, common migraine, tension-vascular headache, tension headache or cluster headache). A clearly defined neurological prodrome was considered to be essential for the diagnosis of classical migraine-eight patients who indicated that visual disturbances began after headache onset were assigned to the common migraine category. The headache history information together with the clinical diagnosis was coded and stored on computer for statistical analysis.

The aim of the first analysis was to assess the objectivity of the clinical diagnoses. Subgroups of headache symptoms that helped separate patients into their clinical categories were identified by the computer using stepwise discriminant analysis. ${ }^{7}$ Because there were five clinical categories, several independent combinations of symptoms (discriminant functions) were needed. To assess the objectivity of clinical diagnoses, each patient was reclassified by the computer to the clinical category with the closest symptom profile. Because clinical diagnoses did not depend on the prevalence of different headache syndromes, no prior adjustment was made for differing group sizes.

The aim of the second analysis was to see whether clinical diagnoses adequately represented the natural grouping of headache symptoms. To ensure that strong relationships among certain closely-associated variables would not mask underlying headache dimensions, indices of gastrointestinal disturbance (nausea, vomiting, diarrhoea) and sensory hyperacuity (sensitivity to light, noise or smells) were formed. Similarly, because headache duration, remissions, and the presence of premonitory symptoms and dull headache between more severe attacks depended to some extent upon headache frequency (patients with daily headache could not, by definition, have a dull headache between more severe attacks), these variables were not included in the second analysis. Instead, the time course of headache was represented by the frequency of the patient's most severe headache episodes. Because of further overlap between the variables unilateral-bilateral headache and throbbing-nonthrobbing headache, the variables bilateral headache and nonthrobbing headache were dropped from the analysis. Correlations were then computed between each remaining pair of variables. The 20 variables that correlated strongly $(p<0.001)$ with at least two other variables were selected for further analysis. Factor analysis (the principal factoring technique with iterations followed by (BBLIQUE rotation of factor axes') was used to identify groups of headache characteristics that commonly were reported together. After the factors were identified, scores on each factor were computed for each patient so that a high score represented a close fit between an individual patient's symptoms and the set of symptoms comprising the factor. Analyses of variance and Scheffe's test for multiple comparisons among group means ${ }^{8}$ were used to see whether factor scores differed significantly among the five clinically-defined headache categories.

\section{Results}

Objectivity of clinical diagnoses

Four discriminant functions each provided independent information that helped to separate the five clinical categories statistically $(p<0.001$ for each function). The first, formed by weighting the presence of a neurological prodrome, separated the classical migraine group (fig 1). The cluster headache category was separated from the other four groups on the second function by selectively weighting fre- 
Table 1 Relationship between clinical and computer classification of 600 headache patients

\begin{tabular}{|c|c|c|c|c|c|c|}
\hline \multirow{2}{*}{$\begin{array}{l}\text { Clinical } \\
\text { classification }\end{array}$} & \multirow{2}{*}{$\begin{array}{l}\text { Number } \\
\text { of cases }\end{array}$} & \multicolumn{5}{|c|}{ Computer classification } \\
\hline & & $\begin{array}{l}\text { Classical } \\
\text { migraine }\end{array}$ & $\begin{array}{l}\text { Common } \\
\text { migraine }\end{array}$ & $\begin{array}{l}\text { Tension- } \\
\text { vascular }\end{array}$ & $\begin{array}{l}\text { Tension } \\
\text { headache }\end{array}$ & $\begin{array}{l}\text { Cluster } \\
\text { headache }\end{array}$ \\
\hline $\begin{array}{l}\text { Classical migraine } \\
\text { Common migraine } \\
\text { Tension-vascular } \\
\text { Tension headache } \\
\text { Cluster headache }\end{array}$ & $\begin{array}{r}152 \\
250 \\
84 \\
56 \\
58\end{array}$ & $\begin{array}{r}152 \\
0 \\
0 \\
0 \\
1\end{array}$ & $\begin{array}{r}0 \\
226 \\
10 \\
0 \\
1\end{array}$ & $\begin{array}{r}0 \\
22 \\
59 \\
10 \\
2\end{array}$ & $\begin{array}{r}0 \\
2 \\
14 \\
46 \\
0\end{array}$ & $\begin{array}{r}0 \\
0 \\
1 \\
0 \\
54\end{array}$ \\
\hline
\end{tabular}

quent headaches recurring in bouts. Bilateral headache not associated with nausea was given most weight on discriminant function 3 , to identify tension and tension-vascular headache (fig 1). These two categories formed opposite poles of the fourth function, which was formed by weighting the combination of unilateral and bilateral headache (that is unilateral headache only some of the time), the absence of nausea and the presence of dull headache persisting constantly between more severe attacks. As can be seen from the scales of discriminant functions 1 to 4 (fig 1 ), groups were separated most on functions 1 and 2 .

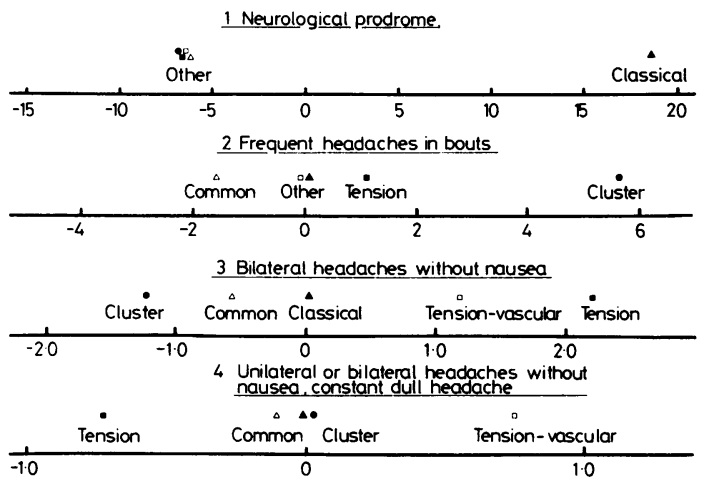

Fig 1 The position of each clinical category on the four discriminant functions. Coefficients greater than $0 \cdot 3$ :

Function 1 - neurological prodrome (1.04)

Function 2 - headache frequency $(0 \cdot 79)$, headache in bouts $(0 \cdot 31)$

Function 3 - Headache without nausea (0.46), headache bilateral $(0.32)$, headache unilateral $(-0.30)$

Function 4 - headache unilateral $(0 \cdot 71)$, constant headache between more severe attacks $(0.51)$, headache without nausea $(0.44)$, headache bilateral $(0 \cdot 33)$.

Note that, by definition, the classical migraine group is separated from the other four groups on the first function. Frequent headache recurring in bouts identifies cluster headache on the second function while groups are spread evenly along the third function. The combination of interictal headache with more severe unilateral or bilateral headache not associated with nausea separates tension-vascular from tension headache on the fourth function.
In the classification phase of the analysis, 537 of the 600 patients were assigned by computer analysis to their clinical category (table 1). Of the $63 \mathrm{mis}-$ classifications, 58 involved the common migraine, tension-vascular and tension headache categories, indicating that these diagnoses were more difficult to make than was classical migraine where the clinical

Table 2 Variables that contributed strongly to the three main factors*

\begin{tabular}{llll}
\hline Variable & \multicolumn{2}{l}{ Factor } & \\
\cline { 2 - 4 } & 1 & 2 & 3 \\
\hline Unilateral pain & 0.44 & & \\
Facial pain & 0.46 & & \\
Running nostril & 0.54 & & \\
Watering eye & $0 \cdot 62$ & & \\
Drooping eyelid & 0.41 & $-0 \cdot 57$ & \\
Headache frequency & 0.41 & $0 \cdot 64$ & \\
Gastrointestinal disturbance & & $0 \cdot 51$ & \\
Sensory hyperacuity & & $0 \cdot 49$ & \\
Facial pallor & & $0 \cdot 42$ & 0.47 \\
Cold hands & & & 0.60 \\
Paraesthesiae & & & 0.54 \\
Dizziness & & & \\
Speech disturbance & & & \\
\hline
\end{tabular}

*Factor loadings greater than $\mathbf{0 . 4}$ are presented.

The magnitude of the factor loading (that is the correlation between the variable and the factor) reflects the importance of the variable for the factor, while the sign of the factor loading shows whether the variable contributes in the same or the opposite direction to other variables.

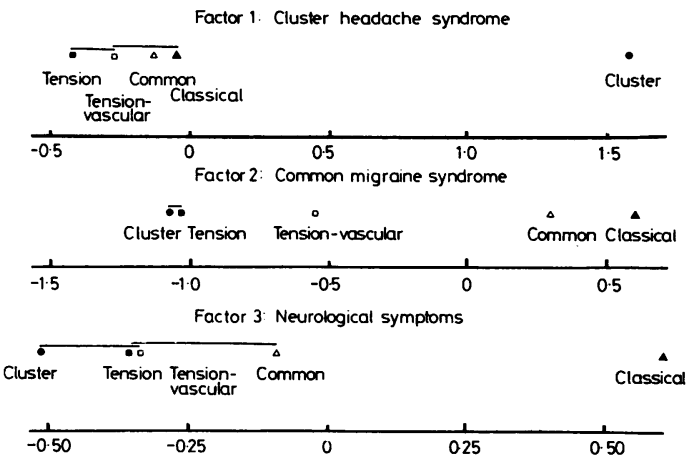

Fig 2 Average factor scores in each clinical category for Factors 1-3. A line above two or more groups indicates that the groups do not differ significantly $(p<0.05)$ from each other by Scheffe's test. 


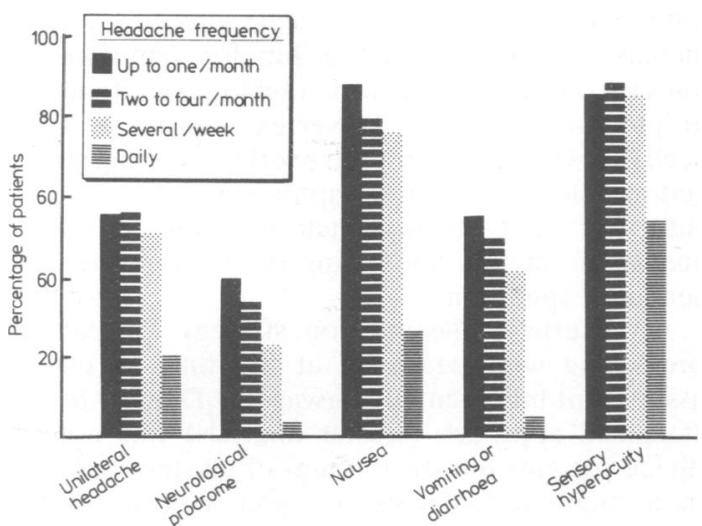

Fig 3 Relationship between the frequency of each patient's most severe headache episodes and migrainous characteristics. Note that the percentage of patients with each feature decreases significantly as headache frequency increases (Kendall's rank order correlation coefficient $=-0.16$ to $-0.31, p, s<0.001)$. Up to one headache per month, $N=95$; two to four headaches per month, $N=229$; several headaches per week, $N=144$; daily headache, $N=74$.

and computer diagnoses agreed in every instance. All but four cluster headache patients were correctly classified by the computer. One of these patients was assigned by computer analysis to the classical migraine category because headaches sometimes were preceded by visual disturbances. Another was misclassified as common migraine because headache recurred only three times per week and was associated with nausea and vomiting, while a further two cluster headache patients were assigned to the tension-vascular category by the computer apparently because a dull ache persisted between severe attacks in one instance and headache recurred every second day in the other. The only other misclassification involved a patient whose headache recurred once or twice per day above the right eye and was assigned to the cluster headache category by the computer. The clinical diagnosis was tension-vascular headache because attacks were not associated with rhinorrhoea, lacrimation or ptosis.

\section{Natural sets of symptoms}

The factor analysis identified five independent sets of intercorrelated variables. The "eigenvalue" for each of the five factors was greater than 1.0 , and together the five factors accounted for $46 \%$ of the total variance shared by the 20 original variables.

The structure of the three main factors is shown in table 2. Since factor loadings for Factors 4 and 5 were greater than 0.4 for only one or two variables, they provided little additional information and were not considered further. Factor 1 consisted of variables that form the cluster headache syndrome, while Factors 2 and 3 were comprised of groups of migrainous variables. Factor 2 resembled the common migraine syndrome in that low frequency (episodic) headaches were likely to be associated with gastrointestinal disturbance, sensory hyperacuity, facial pallor and cold extremities. Factor 3 described an association among symptoms of cerebral disturbance during headache. The presence of a neurological prodrome contributed weakly both to the common migraine syndrome factor (with a factor loading of 0.35 ) and to the neurological symptoms factor (where the factor loading was $0 \cdot 36$ ). Since Factors 2 and 3 correlated strongly with each other $(r=0.44)$, it can be concluded that neurological symptoms before and during headache often were reported together with symptoms of common migraine. By contrast, the cluster headache syndrome (Factor 1) was not correlated with any other factor.

Factor scores were computed for each patient on Factors 1,2 and 3. Analyses of variance demonstrated that all three factor scores differed significantly among the five clinical categories [F,s $(4,595)=44 \cdot 2$ to $181 \cdot 0, p, s<0 \cdot 001]$. Group means and significant differences between each pair of clinical categories are presented in fig 2 . The classical migraine group showed significantly higher average scores than the other four groups on the common migraine syndrome factor and the neurological symptoms factor, whereas the cluster headache category differed significantly from the other four groups on the cluster syndrome factor. The tension and tension-vascular groups differed significantly from the classical and common migraine groups on the common migraine syndrome factor (fig 2).

The relationship between headache frequency, which formed an important part of the common migraine syndrome factor, and migrainous symptoms in the migraine, tension-vascular and tension headache groups is shown in fig 3 . Every migrainous feature was reported less often by patients with daily headache than by patients whose most severe headaches recurred episodically. Furthermore, the percentage of patients who reported gastrointestinal disturbances or a focal neurological prodrome decreased progressively as headache frequency increased (fig 3).

\section{Discussion}

The results demonstrated that almost $90 \%$ of headache patients referred for neurological assessment could be assigned systematically to one of five clinically-defined headache categories. The straight- 
forward definition of classical migraine (headache preceded at least some of the time by a clearlydefined neurological prodrome) ensured that clinical and computer classifications for this category agreed in every instance. By contrast, the overlap between common migraine, tension-vascular and tension headache indicates that current clinical definitions do not separate these categories adequately.

Screening variables for redundancy and analysing only those variables sharing something in common with at least two other variables resulted in a simpler factor solution than has been reported previously. ${ }^{4-6}$ Symptoms of cluster headache formed an independent, clearly-definable syndrome, supporting the view that cluster headache should be regarded as a distinct entity. Neurological symptoms during headache and features of common migraine formed two separate factors, although the strong correlation between these factors indicated that many patients reported both (or neither) sets of symptoms.

The analysis of factor scores demonstrated that the classical migraine, common migraine, tensionvascular and tension headache categories were spaced at regular intervals along the common migraine syndrome factor. As such, they represented differences in the number, rather than the nature, of common migraine symptoms. The tension headache category, for instance, was distinguished by few or no symptoms, while tension-vascular headache provided the link between tension headache and common migraine. Although neurological symptoms during headache were most prevalent in the classical migraine category, this group also reported most common migraine symptoms. Since migrainous features, particularly gastrointestinal disturbances and the presence of a neurological prodrome, decreased progressively as headaches increased in frequency, the present analysis indicates that episodic headaches with many migrainous characteristics may form one end of a headache spectrum, the other extreme consisting of unremitting headaches with few or no features of migraine.

Whether the two extremes of this migrainetension headache spectrum are different clinical entities remains an open question. In his epidemiological study of headache prevalence in a Welsh community, Waters ${ }^{9}$ calculated that the association of three migrainous features (unilateral pain, gastrointestinal disturbance and "warning") was only slightly greater than would be expected by the coincidence of randomly distributed symptoms. There was a far stronger association between the number of patients who reported any one of the three migrainous features and the rating of the severity of the headache. In other words, as the severity rating increased, the number of migrainous symp- toms associated with the headache showed a parallel increase. A similar relationship for symptoms of muscle contraction headache (aching pain, tightness or pressure in the neck, vertex, occiput or forehead), ${ }^{10}$ with considerable overlap of migrainous and muscle contraction symptoms in the same individuals, ${ }^{1{ }^{10}}$ suggests that headache severity could be one of the factors underlying the migraine-tension headache spectrum.

An alternate classification strategy for patients presenting with headache at the time of clinical assessment has been put forward by Diehr et al. ${ }^{11} \mathrm{~A}$ statistical approach (cluster analysis) was used to divide patients into two groups or clusters, resulting in a migraine-like category and a cluster which resembled tension headache. Because there are no objective criteria for determining the number of clusters of patients within a given population, further subgroups of patients were formed until there were eight separate clusters. At this point, the classical migraine category contained only $8 \%$ of patients. Other categories included patients with almost all or very few symptoms, rapid onset headache, symptoms of tension headache, eye pain or symptoms of systemic or upper respiratory infection. When grouped in this way, some of the categories suggested specific alternate treatments (eye examinations, counselling or antibiotics) for patients who might have been diagnosed as having migraine headache. This approach would appear to be more useful in casualty settings than in neurological consultation where patients usually do not present with an acute headache at the time of the clinical interview.

It can be concluded that clinically-defined headache categories (with the exception of cluster headache) represent different points in a continuum rather than being discrete entities. Whether a single common mechanism underlies this headache spectrum, or whether two or more mechanisms interact to produce head pain can be determined only by further studies of the pathophysiology of headache.

This project was generously aided by the JA Perini Family Trust and Sandoz AG (Basel). The figures were photographed by the Medical Illustration Unit, University of New South Wales.

\section{References}

${ }^{1}$ Diehr P, Wood R, Wolcott B, Slay L, Tomkins RK. O the relationships among headache symptoms. . Chronic Dis 1982;35:321-31.

${ }^{2}$ Diehr P, Wood RW, Barr V, Wolcott B, Slay L, Tomkins RK. Acute headaches: presenting symptoms and diagnostic rules to identify patients with tension and 
migraine headache. J Chronic Dis 1981;34:147-58.

${ }^{3}$ Ad Hoc Committee on Classification of Headache. Classification of headache. JAMA 1962;179:717-8.

${ }^{4}$ Ziegler DK, Hassanein R, Hassanein K. Headache syndromes suggested by factor analysis of symptom variables in a headache prone population. J Chronic Dis 1972;25:353-63.

${ }^{5}$ Ziegler DK, Hassanein RS, Couch JR. Headache syndromes suggested by statistical analysis of headache symptoms. Cephalalgia 1982;2:125-34.

- Peck DF, Attfield ME. Migraine symptoms on the Waters Headache Questionnaire: a statistical analysis. J Psychosom Res 1981;25:281-8.

${ }^{7}$ Nie NH, Hull CH, Jenkins JG, Steinbrenner K, Bent DH. Statistical Package for the Social Sciences (2nd ed). New York: McGraw-Hill, 1975.

${ }^{8}$ Keppel G. Design and Analysis: $A$ Researcher's Handbook. New Jersey: Prentice-Hall, 1973.

9 Waters WE. The epidemiological enigma of migraine. Int J Epidemiol 1973;2:189-94.

${ }^{10}$ Kaganov JA, Bakal DA, Dunn BE. The differential contribution of muscle contraction and migraine symptoms to problem headache in the general population. Headache 1981;21:157-63.

${ }^{11}$ Diehr P, Diehr G, Koepsell T, Wood R, Beach K, Wolcott B, Tomkins RK. Cluster analysis to determine headache types. J Chronic Dis 1982;35:623-33.

\section{Appendix}

Variables obtained from the headache history form which were used in the statistical analysis.

1. Frequency of headaches (up to $1 /$ month $=1,2-4$ / month $=3$, several $/$ week $=12$, daily $=28$, more than $1 /$ day $=56)$

2. Headache duration (less than 12 hours $=0$, more than 12 hours $=1$ )

3. Headaches in bouts ( 2 or more headaches/week separated by remissions of at least one month)

4. Unilateral headaches

5. Bilateral headaches

6. Constant dull headache between more severe attacks
Site

7. Temple(s)

8. Frontal (one or both sides)

9. Orbit(s)

10. Facial (one or both sides)

11. Occiput (one or both sides)

Premonitory symptoms

12. Elation, irritability, depression, hunger, thirst or drowsiness 1-24 hours before the headache

Prodromal symptoms

13. Visual disturbances, paraesthesiae or other focal neurological symptoms immediately preceding the headache

Associated symptoms

14. Nausea

15. No nausea

16. Vomiting

17. No vomiting

18. Diarrhoea

19. Photophobia

20. Phonophobia

21. Gustophobia

22. Facial pallor

23. Facial flushing

24. Vascular distension in the temple or forehead

25. Throbbing headache

26. Nonthrobbing headache

27. Scalp tenderness

28. Facial swelling

29. Sweating

30. Cold hands or feet

31. Nose blocked or running

32. Eye(s) watering

33. Eyelid(s) drooping

34. Paraesthesiae during the headache

35. Dizziness or ataxia

36. Speech problems (slurring or dysphasia)

37. Faintness

38. Confusion

39. Shortness of breath

40. Visual disturbance during headache 